\title{
Equifacetted 3-Spheres as Topes of Nonpolytopal Matroid Polytopes
}

\author{
J. Bokowski and P. Schuchert \\ Mathematics Department, University of Darmstadt \\ Schloßgartenstraße 7, D-64289 Darmstadt, Germany \\ bokowski@mathematik.th-darmstadt.de \\ schuchert@mathematik.th-darmstadt.de
}

\author{
Manchmal interessieren uns mehr die \\ kombinatorischen Beziehungen zwischen den \\ Bestandteilen eines Polytops als das ganze Polytop, \\ betrachtet als $n$-dimensionaler Körper. \\ László Fejes Tóth, Reguläre Figuren, \\ Das allgemeine reguläre Polytop, S. 128.
}

\begin{abstract}
We investigate simplicial 3-manifolds, in particular 3-spheres, with few vertices such that the links of all vertices are combinatorially equivalent (equilinked 3-spheres), and, simple 3-manifolds, in particular 3-spheres, with few facets such that all facets are combinatorially equivalent (equifacetted 3-spheres).
\end{abstract}

\section{Combinatorial Spheres and Polytopes}

Using the one-point compactification of the euclidean plane via stereographic projection, we consider Fig. 1 as the icosahedral triangulation of the 2-sphere $S^{2}$ with $f_{0}=12$ vertices. According to Steinitz' theorem, see [27], we understand in particular the correspondence between the boundary structure of simplicial 3-polytopes and triangulations of the 2 -sphere $S^{2}$. All combinatorial types of simplicial 3-polytopes can be obtained via the generation of combinatorial types of triangulations of the 2-sphere $S^{2}$ studied as planar pictures. In addition the icosahedral triangulation has isomorphic vertex figures, i.e., the link of each vertex is the same combinatorial 1-sphere, a 5-gon. 


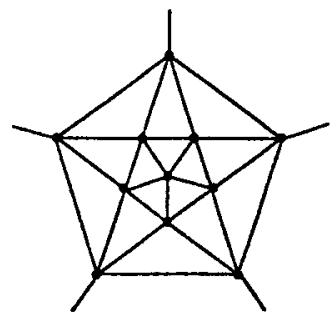

Fig. 1. Icosahedral triangulation of the 2-sphere.

Now the stage is set to use the one-point compactification of euclidean 3-space for studying the combinatorial types of three-dimensional triangulations of the 3 -sphere via spatial pictures. We investigate in particular those finite three-dimensional triangulations (simplicial subdivisions) of the 3-sphere $S^{3}$ such that the link of each vertex forms the same combinatorial 2-sphere. By a simplicial subdivision of the 3-sphere $S^{3}$, we understand a piecewise linear homeomorphism of the boundary of a 4-simplex onto a finite set of $k$ tetrahedra $\left\{T_{1}, T_{2}, \ldots, T_{k}\right\}$ with corresponding identified boundaries. We denote such a simplicial subdivision of the 3 -sphere by its set of tetrahedra $\left\{T_{1}, T_{2}, \ldots, T_{k}\right\}$, and we call it a simplicial equilinked 3-sphere when the face l.ttice of $\left\{T_{1}, T_{2}, \ldots, T_{k}\right\}$ has the property that the link of each vertex forms the same combinatorial 2-sphere.

By introducing the polar concept, see [24], we speak in a similar fashion of a simple equifacetted 3-sphere $\left\{F_{1}, F_{2}, \ldots, F_{n}\right\}$. In this case we have a simple subdivision of the 3-sphere $S^{3}$, i.e., a piecewise linear homeomorphism of the boundary of a 4-simplex onto a finite set of $n$ 3-polytopes $\left\{F_{1}, F_{2}, \ldots, F_{n}\right\}$ exists such that each $F_{i}$ is of the same combinatorial type. Three-dimensional simple equifacetted 3-spheres which do not occur as the boundary of a 4-polytope do exist. In fact, we present such a new example which has Dürer's octahedron, compare Fig. 2, as its facet structure. Starting with isomorphic copies of Dürer's octahedron, we see that by gluing them together, there is a unique way to obtain a simple equifacetted combinatorial 3-manifold. The sphere structure in this case can be established by using oriented matroid theory. The anti-isomorphic face lattice of this 3-manifold occurs as the Las Vergnas lattice of a matroid polytope which confirms the sphere property. Finally, a final polynomial argument shows that this 3-sphere cannot occur as the boundary of a 4-polytope, the matroid polytope is nonpolytopal.

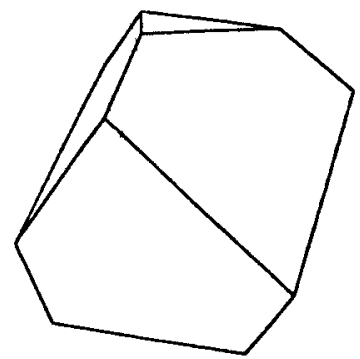

Fig. 2. Dürer's octahedron from his copperplate engraving Melencolia I, 1514. 
Having this result in mind, we are faced with difficulties in this context. An analogue of the above Steinitz' theorem for 2-spheres is missing for 3-spheres. Therefore, it is natural first to study simplicial equilinked 3-spheres or simple equifacetted 3-spheres for their own sake (combinatorial tilings of the 3-sphere) and to come back later to the question which of them are isomorphic to the boundary of a 4-polytope.

Problem 1. Find simplicial equilinked 3-spheres or simple equifacetted 3-spheres.

Some of the four-dimensional analogues of the Platonic solids provide solutions, so we ask in particular:

Problem 2. Can we find infinite series of examples for Problem 1?

Again we find an affirmative answer via classical polytope theory: the boundary structure of cyclic polytopes, or of their polar convex polytopes, respectively.

Problem 3. What about a complete enumeration of these 3-spheres up to a certain number of vertices or facets?

This problem turns out to be more difficult and the following one is even more difficult.

Problem 4. Are these 3-spheres boundaries of convex 4-polytopes?

We have touched upon a long standing area of research. In topology we have the difficult problem of characterizing 3-spheres by certain invariants (Poincaré conjecture). In convexity we have the problem known as the Steinitz problem. We find recent references in the Handbook of Convex Geometry in Mani-Levitska's article [16]. Problem 1 can also be viewed as part of a question about tilings posed by Danzer at the Durham Symposium in 1975, see [15] and [8].

These problems are attractive because of their natural character but we are faced with unexpected difficulties when we search for complete solutions.

Within this article we provide an extended infinite sample class for Problem 2 based on the work of Smilansky, we present a complete enumeration of solutions to Problem 3 with up to 10 facets, and we solve Problem 4 within this class. Our results can also be viewed as a contribution to the theory of tilings of euclidean space, see [23].

One approach for tackling the open Steinitz problem is motivated by corresponding results in matroid theory. The idea is to provide an infinite class, say of simplicial equilinked 3-spheres which are not isomorphic to the boundary of any 4-polytope, whereas after a local change we do have this property. Thus one would have the assertion that there is no local characterization of those 3-spheres which are boundaries of 4-polytopes. Unfortunately, the result of Kalai [13], there are more spheres than boundary complexes of polytopes, is not applicable in the 3-sphere case. Our investigation and the list of matroid polytopes for small numbers of vertices might serve as a starting point for further investigation along this line. 


\section{Three-Spheres Derived from 4-Polytopes}

As mentioned in Section 1 we find solution classes for Problem 1 from classical polytope theory. The regular polytopes with Schläfli symbol $\{3,3,3\},\{3,3,4\},\{4,3,3\}$, $\{3,3,5\}$, and $\{5,3,3\}$ as well as the cyclic 4-polytopes $C_{n}$ and its polar polytopes $C_{n}^{*}$ provide corresponding assertions.

A class of well-studied 3-spheres are the joins of two combinatorial 1-spheres. By taking the join of two $k$-gons we obtain a simplicial 3 -sphere with $k^{2}$ tetrahedra. This sphere has isomorphic vertex figures and a dihedral automorphism group. We denote by $J_{k}$ the 4-polytope whose boundary is the join of two $k$-gons. It turns out that the joins and the boundaries of cyclic polytopes occur as boundaries of bicyclic 4-polytopes of Smilansky [25], [26].

Within this section we define a special class of these bicyclic 4-polytopes. They provide additional solutions to Problem 2 . The vertices of these bicyclic polytopes lie on the 3-torus $S^{1} \times S^{1}$ :

$$
T^{2}:=\left\{(\cos 2 \pi x, \sin 2 \pi x, \cos 2 \pi y, \sin 2 \pi y)|| x \mid \leq \frac{1}{2},\left\{y \mid \leq \frac{1}{2}\right\} \subseteq \mathbb{R}^{4} .\right.
$$

For any pair $(p, q)$ of natural numbers, $p, q \in \mathbb{N}$ with the greatest common divisor of $p$ and $q$ equal to 1 , i.e., $\operatorname{gcd}(p, q)=1$, we have a bicyclic 4-polytope $P_{n, p, q}$ as the convex hull of $n$ vertices $v_{i}, 1 \leq i \leq n$,

$$
v_{i}:=\left(\cos 2 \pi \cdot p \cdot \frac{i}{n}, \sin 2 \pi \cdot p \cdot \frac{i}{n}, \cos 2 \pi \cdot q \cdot \frac{i}{n}, \sin 2 \pi \cdot q \cdot \frac{i}{n}\right) .
$$

By Proposition 2.2. of [26] we know that the bicyclic 4-polytope $P_{n, p, q}$ is simplicial if and only if $p \neq q, \operatorname{gcd}(p, n) \leq 2$, and $\operatorname{gcd}(q, n) \leq 2$. We are interested only in the simplicial case. This implies that we can fix $p:=1$, and we have the conditions $q \neq 1$ and $\operatorname{gcd}(q, n) \leq 2$. Now we abbreviate these simplicial bicyclic polytopes by $P_{n, q}:=P_{n, 1, q}$. The following theorem is a consequence of Smilansky's investigation.

Theorem 2.1. The boundary of a bicyclic 4-polytope $P_{n, q}$ with $q \neq 1, \operatorname{gcd}(q, n) \leq 2$ is a simplicial equilinked 3-sphere. The boundary of the polar polytope $\left(P_{n, q}\right)^{*}$ is a simple equifacetted 3-sphere.

Proof. With the orthogonal matrices

$$
\begin{gathered}
T_{q}(\alpha):=\left(\begin{array}{cccc}
\cos 2 \pi \alpha & \sin 2 \pi \alpha & 0 & 0 \\
-\sin 2 \pi \alpha & \cos 2 \pi \alpha & 0 & 0 \\
0 & 0 & \cos 2 \pi \alpha q & \sin 2 \pi \alpha q \\
0 & 0 & -\sin 2 \pi \alpha q & \cos 2 \pi \alpha q
\end{array}\right) \\
R:=\left(\begin{array}{rrrr}
1 & 0 & 0 & 0 \\
0 & -1 & 0 & 0 \\
0 & 0 & 1 & 0 \\
0 & 0 & 0 & -1
\end{array}\right)
\end{gathered}
$$


we have the relations $v_{i} \cdot T_{q}(1 / n)=v_{i+1}, v_{i} \cdot R=v_{n-i}$, i.e., $P_{n, q}$ has the dihedral symmetry group generated by $T_{q}(1 / n)$ and $R$. Every element of this group induces a combinatorial automorphism on the boundary structure of $P_{n, q}$. The combinatorial automorphism group is vertex-transitive which implies that all vertex figures are isomorphic.

\section{Some Examples and Properties of Bicyclic Polytopes.}

- The bicyclic polytope $P_{n, 2}$ is cyclic.

-The bicyclic polytope $P_{n,(n-1) / 2}$ is cyclic provided $n$ is odd.

- The bicyclic polytope $P_{n, n / 2-1}$ is the join-polytope $J_{n / 2}$ of two $n / 2$-gons provided $n$ is even.

- The bicyclic polytope $P_{n, q}$ is isomorphic to $P_{n, n-q}$.

- The Coxeter-Petrie-polytope which is associated with the polyhedron $\{6,4 \mid 3\}$ in the sense of Coxeter $[7$, p. 78$]$ is isomorphic to a polar of the bicyclic polytope $P_{10,3}$.

- We have shown one example, the polytope $\left(P_{11,3}\right)^{*}$, in Fig. 3 via two complementary orthogonal two-dimensional projections, the edges of one facet are marked bold. Compare these two-dimensional projections with the projections of the Coxeter--Petrie-polytope $\{6,4 \mid 3\}$ which can be found in Coxeter's article [7].

All properties mentioned here are consequences of Smilansky's article [26].

Now already having a huge example class of simplicial equilinked 3-spheres (and by polarity simple equifacetted 3 -spheres), we can ask ourselves whether we have all of them. We provide a complete answer for small numbers of vertices in the following sections.

\section{Generating Simple Equifacetted 3-Manifolds}

We can already find a complete list of combinatorial types of triangulations of the 2-sphere $S^{2}$ representing all simplicial 3-polytopes with vertex numbers $n \leq 9$ in [17]
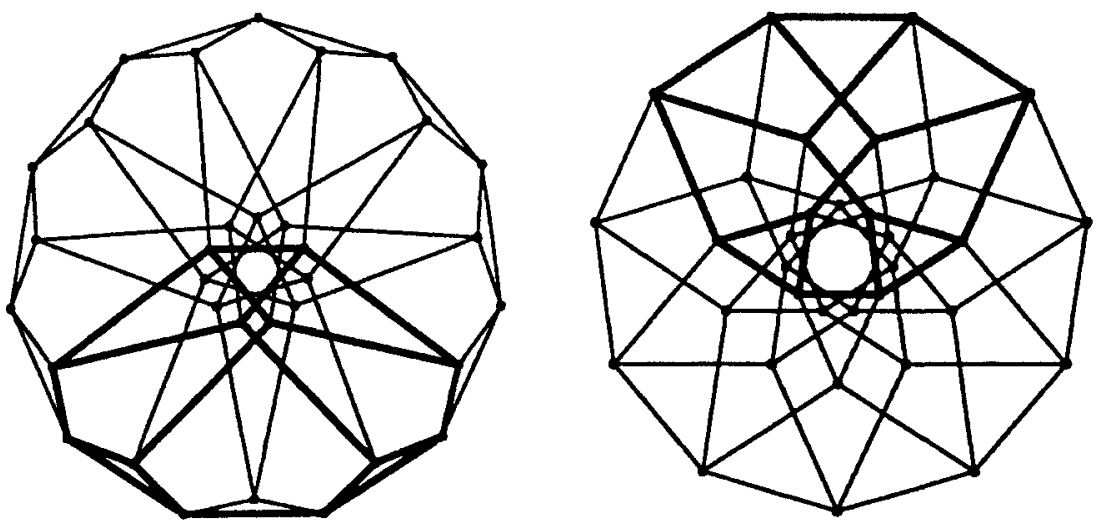

Fig. 3. Complementary projections of the polar bicyclic 4-polytope $\left(P_{11,3}\right)^{*}$. 
and its polar version at the turn of this century in [6] and [12]. The 3-sphere case is more difficult. In order to find a complete enumeration of simple equifacetted 3-spheres with up to 10 facets, we generate all corresponding combinatorial 3-manifolds first. This embedding of the problem is natural. It is possible to confirm the combinatorial 3-manifold property, because we have Steinitz' theorem for 2-spheres. The problem remains to determine the 3-spheres among these 3-manifolds.

First we have to fix some notation. We define a simplicial (combinatoriai) $(d-1)$-sphere (PL-sphere) to be a simplicial $(d-1)$-complex which is homeomorphic (piecewise linear) to the boundary of the $d$-simplex. A simplicial combinatorial $d$-manifold is a simplicial $d$-complex such that the link for each vertex $v, \operatorname{link}(v)$, is a combinatorial $(d-1)$-sphere. We are interested only in the three-dimensional case. Here we define in particular a simplicial equilinked 3-manifold as a simplicial combinatorial 3-manifold with the same links.

We consider also the polar concepts, a simple 2-sphere and a simple equifacetted 3-manifold, i.e., we have a cell complex, the lattice of which is anti-isomorphic to a simplicial 2-sphere and a simplicial equilinked 3-manifold, respectively. We call a simple 2-sphere a facet, or a nonfacet, when it occurs as the boundary structure of a 3-cell in a simple equifacetted 3-manifold, or when it does not, respectively.

In order to generate simple equifacetted 3-manifolds, we use a gluing process of simple 2-spheres which we describe next. Some of such 2-spheres with eight faces are shown by their Schlegel diagram in Fig. 4. We use Brückner's labeling for the 2-spheres [6]. In the first line of the figure we have $B_{1}^{8}, B_{4}^{8}, B_{7 a}^{8}, B_{14}^{9}$, and in the second line we have $B_{12}^{8}, B_{13}^{8}, B_{7 b}^{8}, B_{9}^{8}$.

We call a 2-sphere $S$ a candidate of a simple equifacetted 3-manifold, when we do not know whether it is a facet or a nonfacet. We denote the set of 2-faces of the candidate $S$ by $\left\{F_{1}, \ldots, F_{m}\right\}$. Every edge $e$ of $S$ lies in precisely two 2 -faces $A(e)$ and $B(e)$ and has precisely one vertex in common with two 2-faces $C(e)$ and $D(e)$. We consider for all edges $e$ of $S$ the tuple $\left(\left\{f_{0}(A(e)), f_{0}(B(e))\right\},\left\{f_{0}(C(e)), f_{0}(D(e))\right\}\right)$ where $f_{0}\left(F_{i}\right)$ is the number of vertices of the 2 -face $F_{i}$. The set $K(S)$ contains all such tuples of $S$.
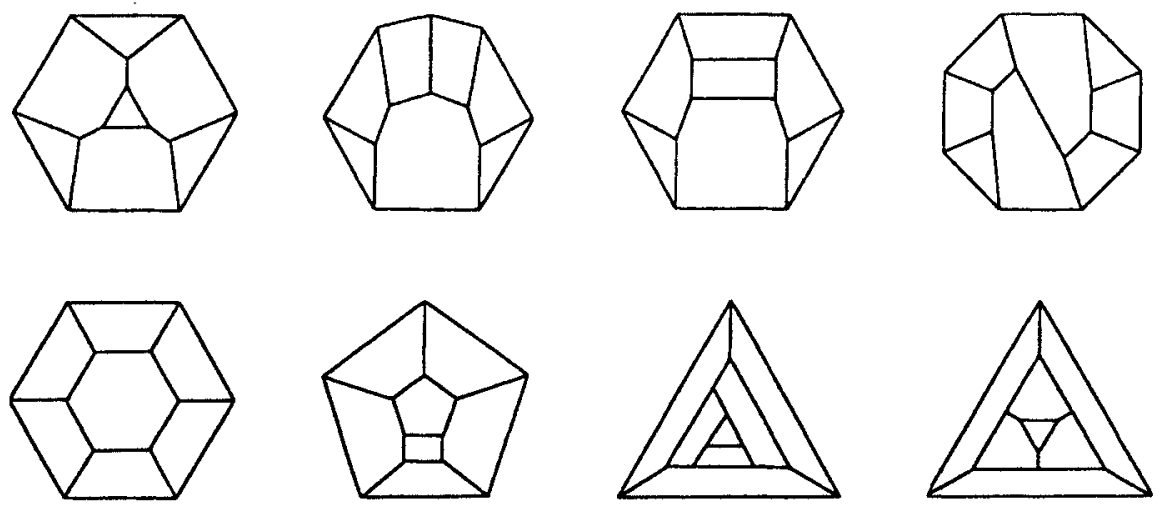

Fig. 4. Some simple 2-spheres with eight and nine faces, facets. 
For a given 2-face $F_{i}$ of $S$ we pick a cyclic ordering of all its adjacent 2-faces $\left(F_{i_{1}}, \ldots, F_{i_{k}}\right)$ of $S, k:=f_{0}\left(F_{i}\right)$, and we define a cyclic neighborhood tuple $v_{k}:=$ $\left(f_{0}\left(F_{i_{1}}\right), \ldots, f_{0}\left(F_{i_{k}}\right)\right)$. If we glue the candidate $S$ with a copy $c(S)$ of $S$, we identify two 2-faces $F_{i}$ and $c\left(F_{j}\right)$ with $l:=f_{0}\left(F_{i}\right)=f_{0}\left(F_{j}\right) . F_{i}$ is a face of our candidate $S$ and $c\left(F_{j}\right)$ is a face of the first copy of $S$. We have to check all combinatorial combinations (cyclic rotations) for gluing our sphere $S$ with its copy. We write down such a combination as a pair of two cyclic neighborhood tuples $\left(\begin{array}{l}\left(f_{0}\left(F_{t_{1}}\right), \ldots, f_{0}\left(F_{t_{1}}\right)\right) \\ \left(f_{0}\left(F_{t_{1}}\right), \ldots, f_{0}\left(F_{21}\right)\right)\end{array}\right)$ for $F_{i}$ in the candidate and $c\left(F_{j}\right)$ in the first copy.

In a simple 3-manifold $M$, every edge $e$ of $M$ lies in three facets. This implies that each edge $e$ of $F_{i}$ must be contained in a further copy of $S$. This copy must have a common 2-face with $S$ as well as with the first copy $c(S)$. Now we look at altogether $l$ additional copies $c_{a}(S), a \in\{1, \ldots, l\}$, of our candidate. The copy $c_{a}(S)$ must contain two adjacent faces $c_{a}\left(F_{x}\right), c_{a}\left(F_{y}\right)$ with $f_{0}\left(F_{x}\right)=f_{0}\left(F_{i_{a}}\right)$ and $f_{0}\left(F_{y}\right)=$ $f_{0}\left(F_{j_{a}}\right)$. This is a first condition which must hold.

Even if it is possible to glue a copy $c_{a}(S)$ at every pair of 2-faces $F_{i_{a}}$ and $c\left(F_{j_{a}}\right)$, we still need to check whether it is globally possible, i.e., whether all $l$ copies of our candidate are compatible together with $S$ and $c(S)$. Therefore we study the combinatorial intersections along the edges of $F_{i}$ between these $l$ copies of $S$. These 2-faces can be ordered in a cyclic manner as $\left(G_{1}, \ldots, G_{l}\right)$. We define a corresponding intersection tuple as $z:=\left(f_{0}\left(G_{1}\right), \ldots, f_{0}\left(G_{l}\right)\right)$. Now we use the set $K(S)$. Our edge $e$ of $S$ is a part (No. $a, a \in\{1, \ldots, l\}$ ) of $F_{i}$. The pair of 2-faces $F_{i_{a}}$ and $c\left(F_{j_{a}}\right.$ ) play the role of $A(e)$ and $B(e), G_{a-1}$ and $G_{a}$ play the role of $C(e)$ and $D(e)$. We are interested in the existence of faces $G_{a-1}$ and $G_{a}$ for all $a \in\{1, \ldots, l\}$, i.e., in the intersection tuple $z$. The combination of elements of $K(S)$ produces admissible intersection tuples or a contradiction.

Now we can give a necessary condition for a simple 2 -sphere to be a facet of a simple equifacetted 3-manifold. When, for at least one 2-face and all cyclic neighborhood tuples of our candidate $S$, there is no possibility of finding an intersection tuple (i.e., $G_{i}$ ), $S$ is a nonfacet. If we have found for all 2 -faces of $S$ intersection tuples, we can try to glue further copies of $S$ together to get a 3-manifold. This gluing process was done by hand calculation.

Example. We prove that the 2-sphere shown as a Schlegel diagram in Fig. 5 (Brückner's No. $B_{8}^{8}$ ) is a nonfacet. For both triangular faces the neighborhood tuple is of the form $(5,5,6)$. We have altogether two essential possibilities of finding a pair of neighborhood tuples: $\left(\begin{array}{c}(5,5,6) \\ (5,5,6)\end{array}\right)$ and $\left(\begin{array}{l}(5,5,6) \\ (5,6,5)\end{array}\right)$.

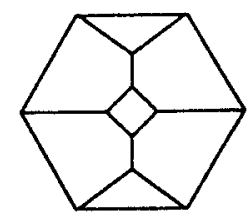

Fig. 5. . A 2-sphere as a Schlegel diagram. 
The first case is not possible when we search for a simple equifacetted 3-manifold because there is only one 6-gon in our given 2-sphere (i.e., there is no element of $K\left(B_{8}^{8}\right)$ with $\left.f_{0}(A(e))=f_{0}(B(e))=6\right)$.

In the second case we have to combine the following elements of $K\left(B_{8}^{8}\right)$ : $(\{5,5\},\{3,4\}),(\{5,5\},\{4,6\})$, and $(\{5,6\},\{3,5\})$. An easy calculation (two subcases) shows that there is no admissible intersection tuple.

By gluing in a systematic manner simple 2-spheres so as to obtain 3-manifolds (hand calculations with computer support) we have found also infinite classes of 3-manifolds.

\subsection{Infinite Classes of Simple Equifacetted 3-Manifolds}

The following infinite classes of simple equifacetted 3-manifolds can be defined by presenting their facets and by the way they have to be glued. The facets are subdivided wedges. The resulting 3-manifolds have a dihedral automorphism group. The number of facets is $n=(s+2)(t-1)+2$ and every facet has $2 t+s-1$ subfacets. Figure 6 describes the facets of these 3 -manifolds $S_{s, t}, s \geq 0, t \geq 3$. The facet of number $n(\equiv 0 \bmod n)$ is shown and the remaining facets are the images under the cyclic symmetry $(1,2, \ldots, n-1, n)$. The labels at all subfacets in the wedges tell us how the facets have to be glued together (vertex numbers to be taken modulo $n$ ). Starting with such wedges the gluing process yields simple equifacetted 3-manifolds.

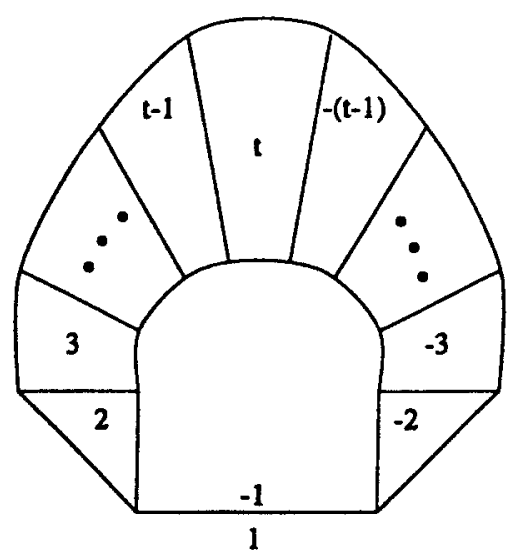

(a)

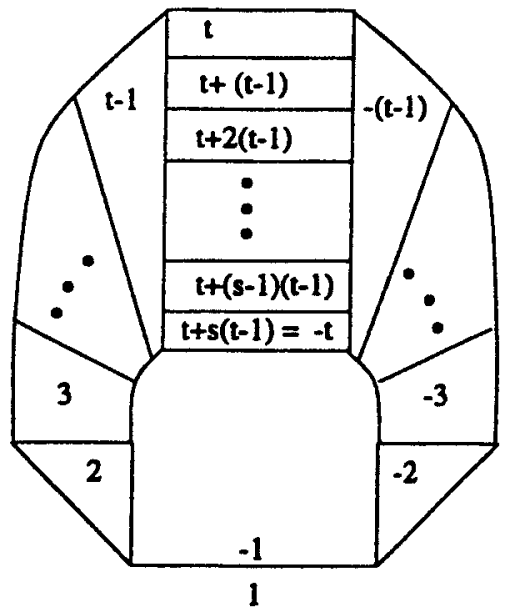

(b)

Fig. 6. Facets of infinite classes of equifacetted 3-manifolds. (a) Wedge for $s=0$ and (b) wedge for any $s$. 


\subsection{Nonspherical 3-Manifolds}

An infinite class of simplicial equilinked 3-manifolds exists, implicitly presented by Kühnel in [14]. This class is a generalization of Kühnel's three-dimensional Möbius-Császár torus. Independent of the number of vertices, each of these 3-manifolds has the same simple 2-sphere as its vertex figure. It is No. $7 \mathrm{~b}$ with eight 2-faces in Brückner's list, see Fig. 4.

The 3-manifold with $n \geq 9$ vertices can be defined by three simplices $(1,2,3,5)$, $(1,2,4,5),(1,3,4,5)$ together with those simplices which we obtain by the required symmetry $\sigma=(1, \ldots, n)$. The permutation $\pi(i)=n+1-i, \forall i \in\{1, \ldots, n\}$, of the vertices defines an additional automorphism, the automorphism group is of dihedral type. All these 3-manifolds are generalizations of the Klein bottle and hence they are not 3-spheres. They can be obtained as the boundary of the 4-manifold defined by the union of the simplex $(1,2,3,4,5)$ with its images under the symmetry of $\sigma$.

\section{Simple Equifacetted 3-Manifolds with up to 10 Facets}

\subsection{Classification of 3-Manifolds}

We return to Problem 3 about complete enumerations of 3 -spheres. Our investigation has led first to a corresponding result concerning 3-manifolds.

Theorem 4.1. There are precisely 17 simple equifacetted 3-manifolds with up to 10 facets.

We have obtained this result by the gluing process of Section 3. We have started with simple 2-spheres of Brückner's classification. A detailed description can be found in Table 1. The numbering of simple 2-spheres is that of Brückner [6].

With 9 faces we have determined 29 nonfacets: Nos. 1, 2, 3, 4a, 4b, 5a, 5b, 7a, 7b, $9 \mathrm{~b}, 10 \mathrm{a}, 10 \mathrm{~b}, 11 \mathrm{a}, 13,15 \mathrm{a}, 17 \mathrm{a}, 17 \mathrm{~b}, 18 \mathrm{a}, 18 \mathrm{~b}, 19 \mathrm{a}, 19 \mathrm{~b}, 19 \mathrm{c}, 22 \mathrm{~b}, 23 \mathrm{a}, 24,25 \mathrm{a}, 25 \mathrm{~b}, 28$, 33. The remaining candidates are not relevant for Theorem 4.1, because Altshuler has classified all simplicial neighborly 3 -manifolds with up to 10 vertices, see [1]. Note that certain 3-manifolds $\left(N_{425}^{10}, N_{3629}^{10}\right.$, and $\left.N_{3631}^{10}\right)$ are not uniquely determined by the type of their facets.

Our investigation has led to one additional simple equifacetted 3-manifold which we have not found in the literature. It has Dürer's octahedron as facet. We study this 3-manifold and certain extensions of it in the next subsections. Especially, we have to show that it is a 3 -sphere.

\subsection{The 3-Manifold $\left(S P_{3}^{10}\right) *$ with Dürer's Octahedron as Facet}

The facets of the simple equifacetted 3-manifold $\left(S P_{3}^{10}\right)^{*}$ are cubes of which a pair of opposite vertices has been truncated. The facet was used by Dürer on his famous copper engraving Melencolia I, see Fig. 2. It has a dihedral automorphism group and 


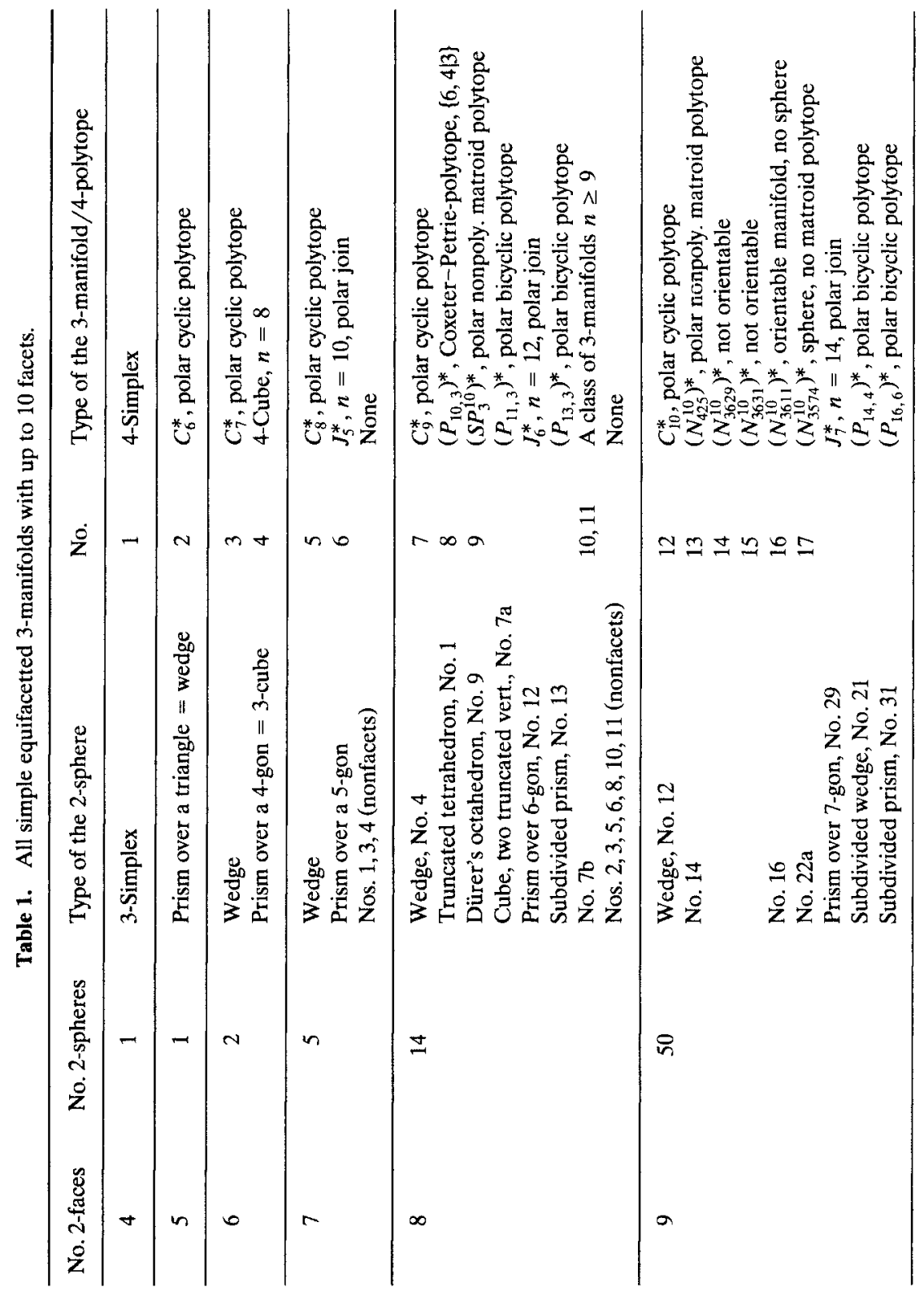




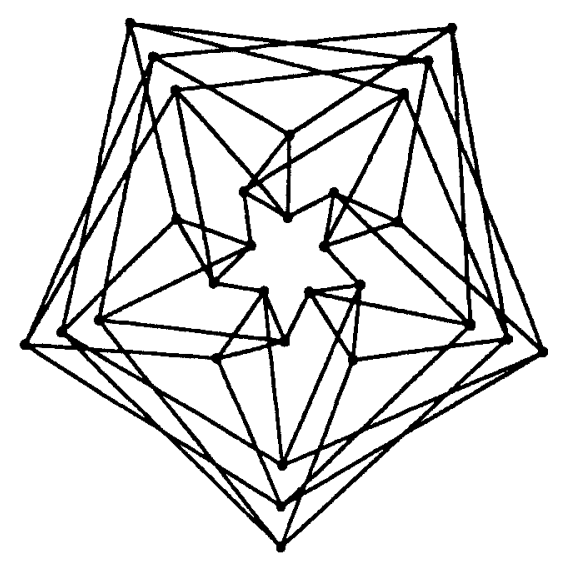

Fig. 7. Edge graph of $\left(S P_{3}^{10}\right)^{*}$.

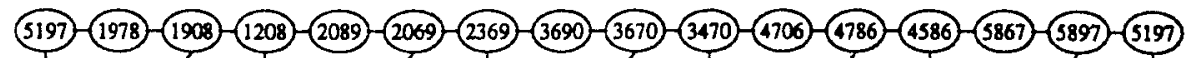

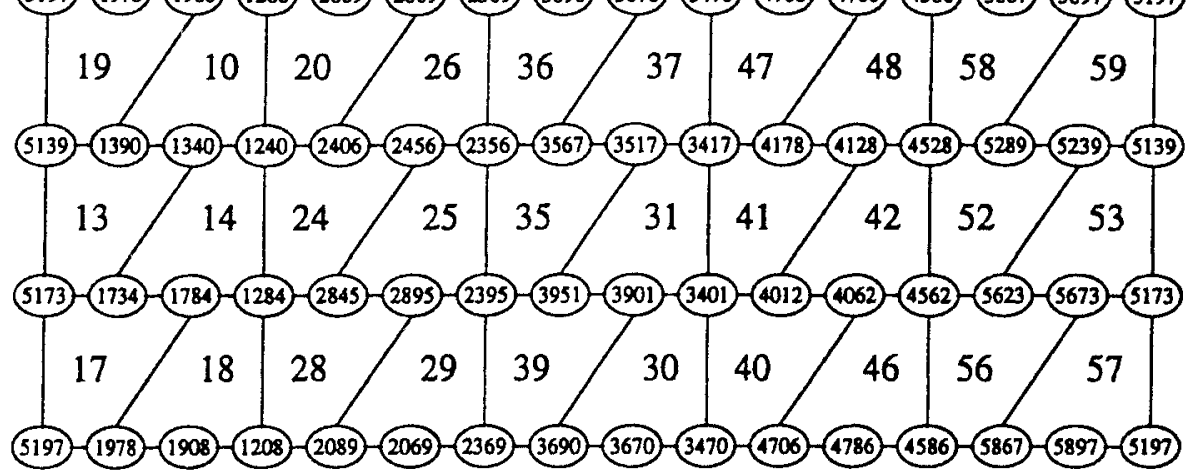

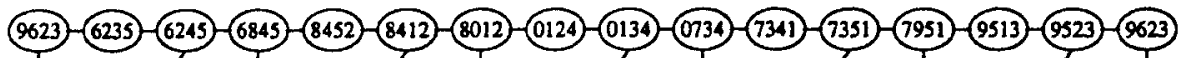

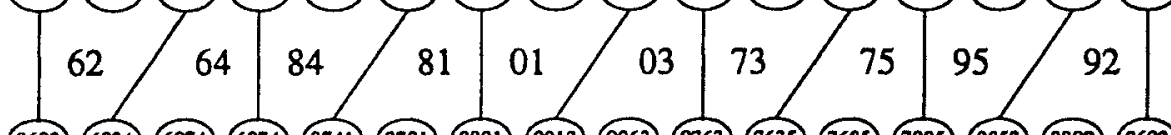

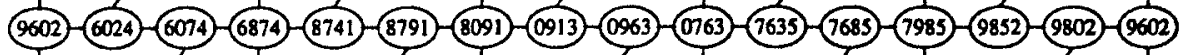

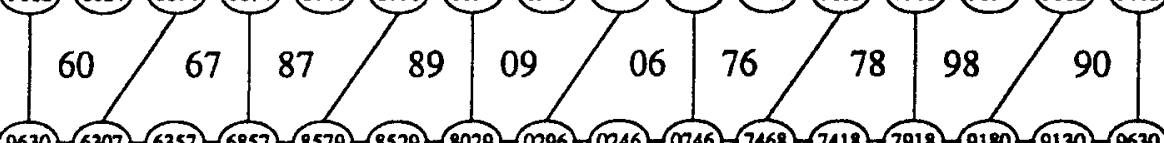

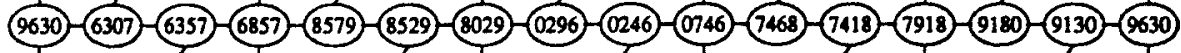

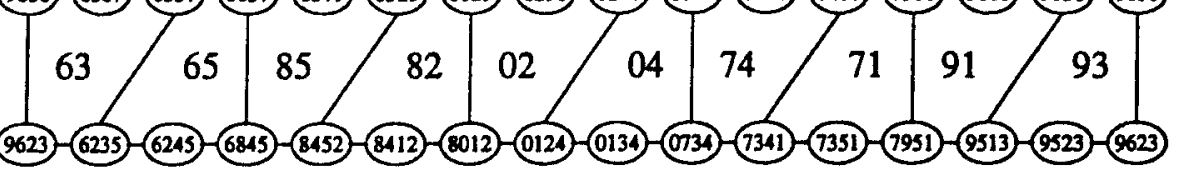

Fig. 8. 3-manifold $\left(S P_{3}^{10}\right)^{*}$, Dürer's octahedron as facet. 
a rotation axis through both triangular faces. We use the term spindle for this facet type, having in mind our extensions in the next paragraph. $\left(S P_{3}^{10}\right)^{*}$ has the vertices $1, \ldots, 9,0$ and it has the symmetries $\sigma=(1,2,3,4,5)(6,7,8,9,0)$ and $\pi=$ $(1,6)(2,8,5,9)(7,3,0,4)$. The automorphism group is of type $Z_{5} \times Z_{4}$. The edges of the 3-manifold $\left(\mathrm{SP}_{3}^{10}\right)^{*}$ are shown in Fig. 7. The combinatorial 3-manifold property (Heegaard-splitting of a 3-sphere) can be confirmed by looking at Fig. 8. Two complementary sets of five facets are shown with their two-dimensional boundaries. The triangles have already been omitted. The additional identification along 2-faces within the upper part (and within the isomorphic lower part) concerns the centerline of 5-gons. The remaining two-dimensional boundary in both cases is a torus with twenty 5-gons. We have a decomposition of this simple equifacetted 3-manifold in two tori. We study this example further in Section 5.

Generalization of $\mathrm{SP}_{3}^{10}$. By generalizing Dürer's octahedron similar to a spindle with arbitrary $n$-gons, we obtain 3-polytopes the first three of which have been shown as Schlegel diagrams in Fig. 9.

This generalization leads to a further simple equifacetted 3-manifold $\left(\mathrm{SP}_{4}^{26}\right)^{*}$. The spindle with two quadrangular faces is a facet of a simple equifacetted 3-manifold with 26 facets. The automorphism group is of type $Z_{13} \times Z_{4}$. We also have a decomposition in two isomorphic tori when we start in a similar way (compare $\left(S P_{3}^{10}\right)^{*}$ ) by first gluing the facets along their quadrangular subfacets. The simplices of $S P_{4}^{26}$ are $(1,2,6,0),(1,2,6, g),(1,2, o, g),(1,2,0, o),(1,6, f, g)$, $(1, f, g, n),(1, g, o, n),(d, e, k, l)$ and the images under the symmetry $(1, \ldots, 0, a, b, c)(d, e, \ldots, o, p)$.

The spindle with two 5-gons is the dodecahedron. It is the facet of the regular 4-polytope $\{5,3,3\}$, the 120 -cell. A result of the theory of Coxeter-groups is that we have a decomposition of the 120-cell in 12 tori (all tori connected by opposite five-gons). To the best of our knowledge, the 120 -cell is the only simple equifacetted 4-polytope whose polar is not within the bicyclic class.

Problem. Are there simple equifacetted 3-manifolds $\left(S P_{k}^{?}\right)^{*}, k>5$, with generalized spindles?

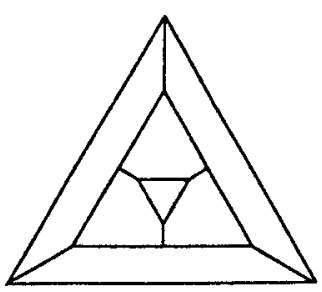

(a)

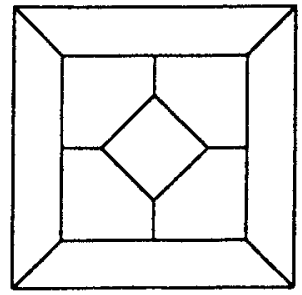

(b)

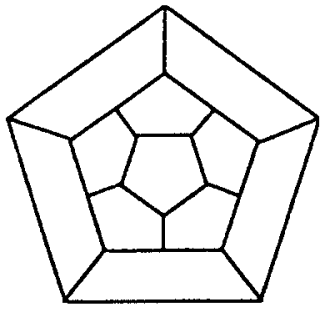

(c)

Fig. 9. Spindle type facets of 3-manifolds. (a) Facet of $\left(S P_{3}^{10}\right)^{*}$, (b) Facet of $\left(S P_{4}^{26}\right)^{*}$, and (c) Facet of $\left(S P_{5}^{120}\right)^{*}$. 


\section{Spheres as Topes of Matroid Polytopes}

In the above sections we have generated simple equifacetted 3-manifolds. Many examples were studied in the literature before. Neighborly 3-manifolds with 10 vertices were studied in [1] and [5]. Boundaries of bicyclic 4-polytopes confirm the sphere property in other cases. For those 3-manifolds where the topological type was known before, the corresponding results are collected in Table 1. The topological type of the 3-manifold $S P_{3}^{10}$ is shown to be a sphere in the last subsection. This leads to the following theorem as an answer to Problem 3.

Theorem 5.1. There are precisely 12 simplicial equilinked 3 -spheres with up to 10 vertices.

All mentioned simple equifacetted 3 -spheres are uniquely determined by the type of their facets.

\subsection{Ordinary Polytopes}

Now we consider Problem 4: whether our spheres can be realized as boundaries of convex 4-polytopes.

Again the above-mentioned collection of articles and the result of the last subsection leads to the following theorem:

Theorem 5.2. There are precisely 9 simplicial equilinked 3-spheres with up to 10 vertices which can be found as a boundary of a 4-polytope. These simplicial equilinked 4-polytopes are isomorphic to bicyclic 4-polytopes.

The 3-manifolds $S_{s, t}$ of Section 3.1 generalize cyclic polytopes ( $s=0$ and $n$ even), they form a class of 3-spheres as boundaries of bicyclic 4-polytopes $P_{(s+2)(t-1)+2, s+2}$.

\subsection{Nonpolytopal Matroid Polytopes}

To decide the open case we use concepts from the theory of oriented matroids, for additional details see [3]. In euclidean space a (convex) $(d-1)$-polytope is defined as a bounded intersection of closed half-spaces given by oriented hyperplanes. Generalizing the oriented hyperplanes to oriented topological hyperplanes with the same intersection properties, we arrive at a pseudohyperplane arrangement. An oriented matroid $\mathscr{M}$ with $n$ elements in rank $d+1$ is a corresponding equivalence-class (PL-homeomorphisms are factored out) of a set of $n$ topological oriented hyperplanes in projective $d$-space. Topes are the cells of maximal dimension in the partition of projective $d$-space by the topological hyperplanes. We call the oriented matroid a matroid polytope, when the boundary of that tope which lies on the positive side with respect to all oriented topological hyperplanes contains a $(d-1)$ dimensional part of each of the $n$ topological hyperplanes. 
This tope has a combinatorial $(d-1)$-sphere as boundary, compare the Folkman and Lawrence Topological Representation Theorem in [3]. The Las Vergnas face lattice $\mathscr{F}_{l v}(\mathscr{M})$ of the matroid polytope is anti-isomorphic to the face lattice of the above tope. An oriented matroid in rank $d$ is uniform when the number of topological hyperplanes intersecting in any point is at most $d-1$. A nonpolytopal matroid polytope $\mathscr{M}$ is a matroid polytope which has a face lattice $\mathscr{F}_{l v}(\mathscr{H})$ which is not polytopal.

A minor minimal matroid polytope is a nonpolytopal matroid polytope $\mathscr{M}$ for which, after any deletion of a vertex $\mathscr{M} \backslash v$, we have a realizable oriented matroid. Minor minimal matroid polytopes are interesting with respect to a negative Steinitztype assertion.

We find in our open case $S P_{3}^{10}$ a matroid polytope (given in terms of signs bases), the tope of which confirms the 3-sphere property. We have found precisely four compatible uniform matroid polytopes with 10 elements of rank 5 . One is presented in the next lines, the others are symmetric images under $\pi$.

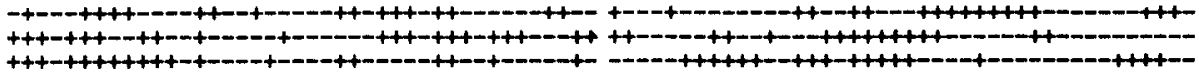

Finally, a final polynomial using two Graßmann-Plücker relations shows that there is no corresponding polytope. The final polynomial reads

$$
\begin{aligned}
0= & \{123 \mid 4569\}+\{124 \mid 3590\} \\
:= & ([12345][12369]-[12346][12359]+[12349][12356]) \\
& +([12435][12490]-[12439][12450]+[12430][12459]) .
\end{aligned}
$$

Because of the symmetry $\sigma=(1, \ldots, 5)(6, \ldots, 9,0)$ and the alternating rule for determinants, this reduces to

$$
0=[12345] \cdot(\underbrace{[12369]}_{>0}-\underbrace{[12490]}_{<0}) \neq 0 .
$$

The three oriented simplices $(2,3,6,9)=+$ and $(1,2,4,0) \stackrel{\pi}{=}(2,3,5,6)=+$ of the 3 -sphere determine the signed bases $[23691]=+$ and $[12409]=+$. This contradiction proves the nonrealizability of the matroid polytope.

We have checked that all deletions of the above matroid polytope are realizable. Together with the neighborly matroid polytope $N_{425}^{10}$, see [5], we have precisely two nonpolytopal simplicial equilinked 3 -spheres with up to ten vertices. $N_{425}^{10}$ can be generated by the seven tetrahedra $(1,2,3,6),(1,2,3,8),(1,2,6,7),(1,2,8,0)$, $(1,3,6,8),(1,6,7,9),(1,6,8,0)$ and its tetrahedra as images of the symmetry $(1, \ldots, 5)(6, \ldots, 9,0)$.

Theorem 5.3. There are precisely two uniform nonpolytopal matroid polytopes with up to ten vertices and isomorphic vertex figures $\left(N_{425}^{10}\right.$ and $\left.S P_{3}^{10}\right)$. They are both minor minimal.

Both nonpolytopal matroid polytopes have isomorphic final polynomials. In fact, this finding was the starting point for the whole investigation. 


\section{References}

1. A. Altshuler. Neighborly 4-polytopes and neighborly combinatorial 3-manifolds with ten vertices. Canad. J. Math. XXIX(2) (1977), 400-420.

2. A. Altshuler, J. Bokowski, and L. Steinberg. The classification of simplicial 3-spheres with nine vertices into polytopes and nonpolytopes. Discrete Math. 31 (1980), 115-124.

3. A. Björner, M. Las Vergnas, B. Sturmfels, N. White, and G. M. Ziegler. Oriented Matroids. Cambridge University Press, Cambridge, 1993.

4. J. Bokowski. Oriented matroids, in Handbook of Convex Geometry, chapter 2.5, pp. 555-602, P. M. Gruber and J. M. Wills, eds., Elsevier, North-Holland, Amsterdam, 1993.

5. J. Bokowski and K. Garms. Altshuler's sphere $M_{425}^{10}$ is not polytopal. European J. Combin. 8 (1987), 227--229.

6. M. Brückner. Vielecke und Vielfache. Teubner, Leipzig, 1900.

7. H. S. M. Coxeter. Regular skew polyhedra in three and four dimension, and their topological analogues, in Twelve Geometric Essays, pp, 75-105. Southern Illinois University Press, Carbondale, IL, 1968. Reprint from Proc. London Math. Soc. (2) 43 (1937), 33-62.

8. L. Danzer, B. Grünbaum, and G. C. Shepard. Does every type of polyhedron tile three-space? Structural Topology 8 (1983), 3-14.

9. L. Fejes Tóth. Reguläre Figuren. Akadémiai Kiadó, Budapest, 1965.

10. B. Grünbaum. Convex Polytopes. Interscience, London, 1967.

11. B. Grünbaum, P. Mani-Levitska, and G. C. Shepard. Tiling three-dimensional space with polyhedral tiles of a given isomorphism type. J. London Math. Soc. 29(2) (1984), 181-191.

12. O. Hermes. Die Formen der Vielflache. J. Reine Angew. Math. 120 (1899) 27-59.

13. G. Kalai. Many triangulated spheres. Discrete Comput. Geom. 3 (1988), 1-14.

14. W. Kühnel. Higher-dimensional analogues of Császár's torus. Resultate Math. 9 (1986), 95-106.

15. D. G. Larman and C. A. Rogers. Durham symposium on the relations between infinite-dimensional and finitely-dimensional convexity. Bull. London Math. Soc. 8 (1976), 1-33.

16. P. Mani-Levitska. Characterizations of convex sets, in Handbook of Convex Geometry, chapter 1.1, pp. 19-41, P. M. Gruber and J. M. Wills, eds., Elsevier, North-Holland, Amsterdam, 1993.

17. T. Oda. Convex Bodies and Algebraic Geometry, Vol. 3. Ergebnisse der Mathematik und ihre Grenzgebiete, No. 15. Springer-Verlag, Berlin, 1988.

18. M. A. Perles and G. C. Shepard. Facets and nonfacets of convex polytopes. Acta Math. 119 (1967), 113-145.

19. E. Schröder. Dürer Kunst und Geometrie. Birkhäuser Verlag, Basel, 1980.

20. E. Schulte. Nontiles and nonfacets for the euclidean space, spherical complexes and convex polytopes. J. Reine Angew. Math. 352 (1984), 161-183.

21. E. Schulte. Tiling three-space by combinatorially equivalent convex polytopes. Proc. London Math. Soc. 49(3) (1984), 128-140.

22. E. Schulte. The existence of non-tiles and non-facets in three dimensions. J. Combin. Theory Ser. A 381 (1985), 75-81.

23. E. Schulte. Generating combinatorial complexes of polyhedral type. Trans. Amer. Math. Soc. 309(1) (1988), 35-50.

24. E. Schulte. Tilings, in Handbook of Convex Geometry, chapter 3.5, pp. 899-932, P. M. Gruber and J. M. Wills, eds., Elsevier, North-Holland, Amsterdam, 1993.

25. Z. Smilansky. Convex hulls of generalized moment curves. Israel J. Math. 52 (1985), 115-128.

26. Z. Smilansky. Bi-cyclic 4-polytopes. Israel J. Math. 70 (1990), 82-92.

27. E. Steinitz and H. Rademacher. Vorlesung über die Theorie der Polyeder. Springer-Verlag, Berlin, 1934.

Received January 18, 1994, and in revised form July 22, 1994. 